\title{
La universidad en la cárcel: expandir los sentidos del trabajo de las personas detenidas
}

\section{The university in prison: expanding the detainees' senses of work}

\section{Rosana E. Sosa*}

* Doctora en Ciencias Sociales, Facultad de Ciencias Sociales, Universidad de Buenos Aires. Magíster en Economía Social, Instituto del Conurbano, Universidad $\mathrm{Na}$ cional General Sarmiento. Licenciada y profesora en Comunicación Social, Facultad de Ciencias Sociales, Universidad Nacional del Centro (FCS, UNC), donde es investigadora y docente exclusiva ordinaria en carreras de Comunicación Social y profesorados de Comunicación Social y Antropología Social. Integrante del Núcleo de Investigación Proincomsci e Ifipraced.

$\triangle$ rosanaesosa@gmail.com http://orcid.org/0000-00021810-5420

\section{RECIBIDO: 11.1.2021}

ACEPTADO: 19.4.2021

\section{Resumen}

El artículo se ocupa de sistematizar una intervención universitaria destinada a promover mapeos colectivos de saberes y experiencias laborales en unidades penitenciarias de la zona centro de la provincia de Buenos Aires. Se trata de un relevamiento en el marco del proyecto de investigación orientado «Acceso a derechos y vida cotidiana en la cárcel» (2019), que problematiza las acciones universitarias destinadas al fortalecimiento de la formación ciudadana plena de personas privadas de libertad.

Palabras clave: educación de los presos, trabajo, prisión, universidad, derechos civiles, Argentina.

\section{Abstract}

The article deals with systematizing a university intervention aimed at promoting collective mapping of knowledge and work experiences in Penitentiary Units in the central area of the province of Buenos Aires. The study, carried out within the framework of the Oriented Research Project «Access to rights and daily life in prison» (2019), makes an issue of university actions aimed at strengthening the full citizen education of people deprived of liberty.

Keywords: correctional education, labour, prisons, universities, civil and political rights, Argentina. 


\section{Introducción}

Este trabajo se inscribe en la tarea de interrogarse y producir conocimiento acerca de las intervenciones socioeducativas de la universidad en materia de fortalecimiento de la ciudadanía plena de personas privadas de la libertad. El interés específico está centrado en el análisis de los modos en que las condiciones de acceso a la educación universitaria y la permanencia en ella constituyen una puerta de entrada para un acercamiento tanto a actividades artísticas y culturales cuanto a un proyecto laboral.

Lo que se presenta en estas páginas son resultados parciales de una investigación en curso de alcances más amplios acerca de los sentidos que adquiere el trabajo en el contexto de las prisiones. Más concretamente, interesan las modalidades que asume la circulación de sentidos sociales asociados al trabajo en el ámbito de la cárcel. Las unidades de estudio (Guber, 1991) son los centros universitarios de tres de las nueve unidades carcelarias que integran el Complejo Penitenciario Centro, uno de los diez que componen la provincia de Buenos Aires. Se trata de las unidades 2 y 38 de la localidad de Sierra Chica, del partido de Olavarría, y la Unidad 52 de la localidad de Azul. En particular, se trabaja con detenidos y detenidas que transitan por alguno de los niveles del sistema educativo y que, por tanto, participan en actividades nucleadas o motorizadas por el Programa Universidad en la Cárcel de la Unicen y los centros universitarios Rodolfo Walsh, de la Unidad Penal 2 de Sierra Chica; Manuel Belgrano, de la Unidad Penal 38 de Sierra Chica, y ALMA, de la Unidad 52 de Azul. Si bien la investigación, por requerimientos metodológicos, es necesariamente localizada, se espera que las conclusiones obtenidas permitan su transferencia a otros contextos similares.

La investigación se desarrolló en distintas etapas durante el año 2018, a partir de un enfoque cualitativo que conduce a la comprensión relacional, histórica y situada de los procesos y prácticas sociales estudiados. El análisis considera fuentes primarias (entrevistas a informantes clave y registros) y fuentes secundarias (documentales y periodísticas que conforman un corpus correspondiente al período 2017-2019 que privilegia los medios de comunicación de las ciudades de Olavarría y Azul).

La construcción de los datos partió de un trabajo etnográfico que incluyó entrevistas en profundidad y registros de observación con participación de acciones de promoción de mapeos colectivos materializados en una secuencia de talleres participativos con fines de relevamiento.

\section{La producción de sentidos del trabajo}

La investigación apunta al análisis de los modos en que acontecen la producción, la circulación y la apropiación de discursos que confluyen en definiciones acerca del 
«trabajo en la cárcel». En particular, resulta de interés el proceso por el cual los estudiantes universitarios detenidos definen acciones que buscan atender tanto a los requerimientos de la justicia cuanto a las representaciones colectivas que remiten al proyecto laboral.

La preocupación que originó esta investigación aspiraba a desentrañar problemas que remiten al plano de la discursividad social (Verón, 1987) como el ámbito en que acontece la producción de relatos y narrativas que se constituyen tanto en estrategias de sectores hegemónicos para favorecer sentidos dominantes que remiten a la cárcel como en tácticas de los actores particulares para habitar en ella cotidianamente.

La relevancia que adquiere la dimensión simbólica y de construcción de significados va acompañada de una atenta mirada de las condiciones histórico-materiales en que esos significados son producidos, reproducidos y disputados. En este marco, es vital la recuperación de los estudios históricos, comunicacionales y culturales de la Escuela de Birminghan. En particular, la concepción de la cultura de Raymond Williams (1982), pensada como entramado compuesto por representaciones simbólicas, prácticas e instituciones que se producen, reproducen y circulan en la sociedad subrayando las formas dinámicas en que diferentes relaciones e intereses — de dominación y de subordinación- coexisten.

La construcción de sentidos del trabajo constituye los modos en que este es tematizado —en los términos propuestos por Habermas (1987) - en el discurso social, focalizando en los factores estructurales que contribuyen a la emergencia de actores, discursos y significados, al tiempo que abrevan en el pasado, lo redefinen y resignifican. Nuestro interés radica en los modos en que estos procesos procuran decirse, ponerse en palabras, contarse - y silenciarse- Reviste especial interés para este trabajo acceder a la opacidad y la negatividad (Lourau, 1988) de los modos en que se proyecta e instrumenta el trabajo en la cárcel.

La consideración de los discursos sociales como ámbito propicio para problematizar el trabajo en la cárcel e indagar allí los sentidos que habitan y significan parte del supuesto de que los modos del decir, lejos de ser un mero reflejo que viene a dar expresión a una realidad material, constituyen un registro social activo que materializa y comunica el dinamismo de la cultura de una sociedad determinada y cómo esta cultura es vivida por esta sociedad. En este punto, la perspectiva del materialismo cultural en el que se encuadran los aportes de Raymond Williams, al tiempo que logran alejarse de la vinculación determinista entre los sujetos y la sociedad, plantea una concepción del lenguaje como «una experiencia activa y cambiante; una presencia social dinámica y articulada dentro del mundo» (Williams, 1982, pp. 50-51). En particular, la idea de estructura de sentimiento aportada por Williams nos introduce a la posibilidad de pensar aquellos significados y valores tal como se viven y se sienten activamente. El interés radica en aquellos significados y valores tal como son vividos y sentidos activamente, 
en las relaciones existentes entre ellos y en las creencias sistemáticas o formales. Dichos aportes conceptuales facilitan la comprensión de los sentidos que interesa analizar.

Al mismo tiempo, acudimos a explorar los imaginarios y prácticas sociales (Baczko, 1991) que convergen en la cultura organizacional (Abravanel, 1992) de la cárcel, entendiendo que es allí donde se exhibe una disputa de sentidos asociados al trabajo. La complejidad de los saberes que se producen en el marco del trabajo y la interrelación que tiene lugar en el curso de la acción son abordados atendiendo a la distinción entre conocimientos, saberes de las acciones y saberes de las situaciones propuesta por Barbier (1996). La categoría saberes del trabajo y la mencionada tipología permiten advertir los matices que en este constructo tienen lugar y dan un salto cualitativo respecto de concepciones sustancialistas que circunscriben una relación social a una enumeración de calificaciones puestas en juego por las personas en el desempeño de un empleo particular.

\section{El escenario de la prisión}

Ni la educación ni el trabajo son actividades que congregan a la mayoría de las personas detenidas, aun cuando estos son los conceptos llave del paradigma de la reinserción social que asume la política criminal del Estado argentino. He aquí una de las tensiones que aparecen reveladas con una rápida mirada de las estadísticas oficiales en materia carcelaria. Allí es posible constatar que ocho de cada diez recluidos (de un universo total de 37.536 personas) no participan en actividades laborales y cinco de cada diez no intervienen en ninguna propuesta formativa (Ministerio de Justicia y Derechos Humanos, 2018).

Algunos datos completan la situación: «Gran parte de los detenidos alcanzan por primera vez el cumplimiento del derecho a la educación estando en la cárcel» (Umpiérrez, 2013). Una historia acumulada de escasas oportunidades de acceso a la cultura —en sentido amplio - y a la educación —en términos particulares - se expresa en trayectorias escolares inconclusas e interrumpidas. Los datos se vuelven incontrastables cuando se traducen en cifras: del total de los recluidos en Argentina, solo un $40 \%$ tiene el primario completo, apenas el $7 \%$ ha terminado su secundario y el $2 \%$ finalizó sus estudios terciarios/universitarios.

Las universidades públicas han ganado presencia en las cárceles desde la recuperación de la democracia en Argentina, y esto es inescindible de la concepción rupturista de la educación con la que fundan su intervención. Lejos de concebirla como un medio para lograr o acreditar la resocialización, la educación se asume como un fin en sí mismo. Su carácter de derecho humano, al tiempo que ensancha su significado, conduce 
a un accionar definido por intervenciones que generan condiciones materiales y simbólicas en las que sea posible gozar este derecho en el encierro.

Pensar el trabajo en la cárcel requiere similares movimientos: reconocer un complejo entramado de sentidos y elucidar los constructos que les subyacen. Tales propósitos son tributarios de una hipótesis que tiene, a la vez, una intención propositiva: los sentidos que adquiere el trabajo están restringidos a un dispositivo destinado a garantizar la reinserción social del criminal. Algo similar acontece en la educación.

\section{El trabajo en la cárcel: imágenes, sentires y prácticas}

El trabajo en las sociedades occidentales capitalistas es parte de una racionalidad que se estructura en torno de su articulación con la educación. La credencial educativa que es otorgada por el Estado asume el carácter de moneda de cambio para obtener un empleo remunerado en el mercado de trabajo (Carton, 1985). Es un sendero que ha sido prolijamente diseñado, pero que no es transitado por todos. Tanto es así que la mitad de la población detenida en las cárceles argentinas no registra ninguna profesión u oficio al momento de ingresar a prisión (53\%) y una proporción similar (49\%) se asienta como «desocupado» (Ministerio de Justicia y Derechos Humanos, 2019).

En este marco, en la calle, el trabajo para quienes están detenidos tiene un carácter esquivo y lejano, mas no difuso y carente de significados. En la tumba, por su parte, multiplicidad de prácticas significan el trabajo y lo constituyen en algo concreto y, por tanto, con notable incidencia en la conformación subjetiva de las personas privadas de libertad.

Una somera exploración en los imaginarios que convergen en la cultura organizacional de la cárcel exterioriza una disputa de sentidos asociados al trabajo. Unos sentidos arraigados que sentencian que el preso tiene que trabajar albergan sentidos de castigo o de terapia y contribuyen a dotar al trabajo de una impronta meritocrática con que los detenidos consiguen acreditar «conducta» $y$, por tanto, acceder a mejoras en sus causas judiciales o bien participar en intercambios informales que pueden mejorar sus condiciones de vida en el encierro.

¿Qué posibilidades de trabajo existen en la cárcel? Las entrevistas a informantes clave han permitido construir una tipología que por embrionaria no impide componer un cuadro de situación. Una primera opción «laboral» consiste en acceder a los talleres y se vincula con la posibilidad de aprender un oficio. Mientras este es el objetivo central que congrega, es la institución penitenciaria la que percibe el dinero generado por la venta de los productos fabricados en los talleres y la que lo destina al mantenimiento de 
las instalaciones o a la creación de nuevos talleres en las unidades. Los internos, por su parte, reciben el peculio establecido por la ley 12256 (Argentina, 1999).

Otra posibilidad «laboral» son las tareas de higiene y mantenimiento de las instalaciones. Los internos que las realizan obtienen un estipendio mensual cuyo valor varía según la actividad y el tiempo en los distintos puestos. Este peculio escasamente representa alguna incidencia en la mejora de las condiciones de vida en la detención y muy frecuentemente es transferido a las familias para financiar sus visitas desde ciudades alejadas de las unidades penales, mayormente en el conurbano bonaerense.

Una tercera posibilidad es identificada en el trabajo de campo a través de fuentes secundarias, pero no se registraron indicios en las unidades penitenciarias analizadas. Se trata de talleres coordinados por uno o más internos, que reciben el nombre de autogestionados. Comienzan con la presentación de un proyecto a la unidad, el cual se somete a evaluación. La autorización de un espacio de trabajo genera movimientos en los que el/los interno/s deberá/n proveerse de herramientas y materias primas y dar comienzo a la producción. En ese caso, quien produce es quien recibe lo obtenido por su comercialización mediante un mecanismo que no es claramente explicitado.

Más allá del tipo de trabajo que se realice, esto no es una realidad que incluya a toda la población carcelaria que está en condiciones o pretende percibir un peculio. En consecuencia, en materia de acceso y mantenimiento del trabajo en prisión, emerge un complejo sistema de relaciones interpersonales -entre presos y con el personal penitenciario- con tácticas asociadas al «hacer conducta». La confluencia de tales factores, a la vez que restringe el sentido del trabajo al «beneficio», genera un escenario en el que es el detenido quien habrá de ocuparse de demandar su acceso al trabajo, que es un bien escaso, y de generar instancias de negociación.

El campo de disputa que se constituye en torno a significar el trabajo en la cárcel trasciende las rejas. Aun cuando no es el análisis discursivo el propósito del presente artículo, nos detendremos en algunas recurrencias del discurso mediático, con especial referencia a las notas de prensa de la mencionada estructura estatal referidas al trabajo de los detenidos de las unidades penitenciarias. Estos textos, considerados en clave de analizador (Lourau, 1988) de aquello que la institución penitenciaria quiere mostrar, logra componer uno de los sentidos más potentes con que se asocia al trabajo en la prisión. 


\section{El trabajo que «se da».}

\section{Exhibiciones, demostraciones y otros despliegues}

«Todo esto es para alguien que lo necesita en serio». Una expresión enunciada por el área de prensa del Servicio Penitenciario Bonaerense es publicada y, más tarde, replicada por medios de comunicación olavarrienses. Las comillas atribuyen un decir y un reflexionar a un interno de la Unidad Penitenciaria 38, aludiendo a su producción de juguetes didácticos destinados a «los jardines de infantes más necesitados de Bahía Blanca y Olavarría». La referencia se completa con señalamientos de la propuesta de talleres para las y los detenidos que conducen a dicha producción, la tarea de docentes que los llevan adelante y un organigrama de la cárcel que contempla un «área de tratamiento» donde «pueden adquirir nociones [y] herramientas [...] para desenvolverse en el mundo laboral».

En principio, las referencias mediáticas conceden un primerísimo primer plano al destino de la producción. La donación como acción y las instituciones «más pobres» como destino constituyen un parteaguas que logra albergar dos trabajos mostrables: el de la institución penitenciaria en materia de educación y formación para la resocialización y el de las y los detenidos que aprenden trabajando para donar. Es un esquema que, por reiterado, parece generar un recorrido exitoso más allá de las variantes en la producción de juguetes de madera, libros en braille y mobiliario escolar, entre otros codiciados productos. Sustentados en una supuesta detección de saberes que se consideran necesarios para «desenvolverse en el mundo laboral», los talleres y los docentes son ponderados en su aporte destinado a la producción de saberes del trabajo.

La primacía que adquiere el destino filantrópico de la producción de las personas detenidas - al mismo tiempo que colabora con la idea de resocialización como fin último- logra un constructo que omite la presencia de un mercado y, con ello, soslaya discusiones acerca del valor económico del producto y del trabajo invertido en su producción.

En este marco, el trabajo de los detenidos aparece subsumido por la donación de objetos producidos, los cuales, haciendo las veces de ofrenda religiosa, marcan un camino hacia la redención (del prefijo re, 'de nuevo', y émere, 'comprar').

El trabajo no es aquí más que un medio para mostrar que sujetos bajo observación hacen buena letra tutelados por una institución que tiene esa misión. El valor del trabajo queda soslayado por el valor de la ofrenda y ambos soslayan el trabajo realizado por los detenidos. En particular, el trabajo vivo que, en términos de Marx, es aquel «existente como proceso y acto» (Marx, trad. 1973, p. 238), la «fuerza de trabajo puesta en acción» (p. 156) que se opone al producto -equivalente a su vez al trabajo objetivado o trabajo muerto. 
Las prácticas que se dan en la cárcel, en particular aquellas que conducen desde la institución a «dar» el producto del trabajo, ponen en evidencia un vínculo entre quienes tienen la capacidad de «dar» el trabajo y quienes tienen «la palabra» en el espacio público. En ese sentido, se retoma la idea de «dar» asociada al trabajo (Sosa, 2014) y la construcción del estatuto de «dadores de trabajo».

Sin embargo, emergen en la vida cotidiana otros saberes. Elegimos, por ello, poner el foco en las percepciones de actores involucrados como condición necesaria para pensar lineamientos y proyecciones que integren, incorporen o recuperen experiencias exitosas y valiosas para sus protagonistas.

\section{Talleres participativos \\ como estrategia de relevamiento}

Los talleres participativos como estrategia de recolección se sustentan en argumentos de orden conceptual y metodológico. Los primeros encuadran la indagación por caminos que, lejos de circunscribirse a las trayectorias laborales, más bien pretenden recuperar los saberes que son reconocidos como propios. Se atiende al concepto de $s a-$ beres del trabajo (Barbier, 1996) y a la relación social que subyace a las calificaciones que tienen una posición relacional.

La metodología de trabajo, por su parte, privilegia un relevamiento desde escenarios de intercambio y compromiso mutuo con las actividades propuestas, que busca hacer inteligible para el colectivo aquello que se observa y se le acredita tal condición de saber.

En este marco, se trabaja desde una perspectiva de la comunicación entendida y practicada como proceso de intercambio y reciprocidad en el que los sujetos generan un proceso grupal de cooperación y arriban a una construcción que se asume colectiva.

La propuesta pretende delinear una recuperación de los saberes que los sujetos asumen tener e indagar acerca de con quién/es los han aprendido. En todos los casos, se planifica una primera aproximación a través de los sentires y pensares que construyen el relato y la narración de eventos que se juzguen significativos para explicar por qué es un saber y por qué es importante para el sujeto que lo identifica. En virtud de ello, la búsqueda exploratoria conduce a tener una mirada que prioriza - aunque no excluye- los saberes de los detenidos en materia de trabajo.

La pertinencia de la estrategia de recolección está fundada en la posibilidad de ingresar a los sentires que ese saber sostiene e identificar a los otros que están involucrados en su aprendizaje: sujetos, organizaciones, trama de relaciones, etcétera. 
La confección del mapeo permitió acceder tanto a los saberes que son identificados como tales cuanto a la/s persona/s vinculada/s con su aprendizaje. Como producto de ello, se arribó a una primera aproximación de los saberes y de los sentidos que les subyacen y los sostienen para las y los estudiantes detenidos.

\section{Los saberes que se saben. Recuerdos y evocaciones}

Las personas detenidas que participaron en los intercambios han reconocido saberes y los han ubicado en la temprana infancia, vinculados a tramas familiares o de amistad y asociados a la ayuda o la colaboración con otro. Estas evocaciones son identificadas con momentos vitales de aprendizaje y como generadoras de pertenencias significativas para el recorrido vital de sus protagonistas.

Una primera instancia se detiene en una identificación de saberes que remiten a la órbita doméstica («cocinar», «escuchar», «reconocer mis errores») y deja paso a una identificación de espacios laborales. Ambos presentan como recurrencia la vinculación del período de aprendizaje a la mediación de un miembro de la familia, directo o indirecto. Mientras algunos parecen postular la posesión de un oficio, la mayoría menciona saberes que, al tiempo que revisten importancia para ellos y son presentados como significativos, se vinculan a hechos o momentos aislados.

Su evocación tiene una fuerte carga emocional al asociarse a «buenos momentos», «buenas personas» $\mathrm{y}$ «otra vida». A este grupo corresponden aquellos vinculados al cuidado personal y al ámbito doméstico.

«Ahí yo aprendí a...», «Me acuerdo que me enseñaba hasta que me saliera bien», «Recuerdo que [yo] era bueno en eso» y que «me daba maña». Afloran espacios y momentos en los que el sujeto que recuerda está aprendiendo y, al mismo tiempo, hay otro que se recuerda enseñándole.

Los sujetos referentes son nombrables, identificables, y se los percibe unidos con cierta cuerda moral que los ubica en «otra vida», la que tuvo lugar en «la calle». Aun cuando hay una valoración positiva del espacio-tiempo en el que se aprendió, no hay registros de continuidad ni de secuencias que muestren un largo plazo con las que sea posible componer una imagen de trabajo. Mucho menos de trabajo remunerado.

No hay alusiones claras a la obtención de remuneraciones por el trabajo. Los recuerdos los ubican siendo el ayudante de otro, que era el trabajador; colaborando con un otro que ostentaba el rol principal; insertándose en un espacio que tenía «propieta- 
rio». Estas recurrencias son las que hacen pensar que se trata de una participación secundaria, que se explica por la finalidad del aprendizaje y se valora por la generosidad del que comparte el trabajo.

Ahora bien, ¿quiénes son estos sujetos recordados por las y los estudiantes detenidos? El aprendizaje se explica por el vínculo o las relaciones que lo sostienen. Se apela a lo parental: «mi madre», «mi viejo», «un tío con el que me mandaba mi madre», «un polaco amigo de mi viejo».

En segundo término, aparecen las tramas institucionales, que remiten casi directamente a «la escuela» o «mi barrio» y que aluden a aprendizajes que, al mismo tiempo que difieren del ámbito familiar y hogareño, lo vinculan por su momento en el ciclo vital. Es la temprana infancia que terminó y que fue sucedida por otra etapa en la que estos episodios no abundan. 0 no existen.

Y, en tercer lugar, aparece la cárcel como ámbito en que el aprendizaje ha tenido lugar, con mayor o igual importancia que algunos de los anteriores. Así, el terreno de la cárcel cristaliza las mismas claves: acreditar un espacio de aprendizaje, valorarlo como experiencia de instrucción, mas prescindir de la remuneración en los términos que corresponderían a un salario.

Los talleres promovidos por la penitenciaría también son rescatados como instancias de aprendizaje. En la misma clave que la escuela, se avizoran como espacios altamente valorados por los internos por ser instancias asociadas a «hacer algo»o "aprender algo». Sin embargo, es una cuestión de orden estructural lo que está pesando en la puesta en valor de las instancias: ser una cucarda mostrable y validada para un mejoramiento en la causa. Aquí la tensión entre ser un sujeto en libertad y ser un sujeto autónomo más allá del contexto (de calle o de encierro) se advierte como una cosmovisión que habrá de atravesar gran parte de nuestros proyectos.

El dato saliente aquí no es el tipo de saberes que exhiben las y los detenidos dado que son diversos-, sino el carácter coyuntural que han tenido en su trayectoria vital. No hay ausencia de recuerdos y evocaciones, sino más bien abundancia de episodios, pero estos se expresan aislados y estrictamente ubicados en «momentos vividos» asociados al trabajo.

Un segundo apunte que se advierte está relacionado con la fragmentación que evidencian los aprendizajes en la cárcel, los cuales, aunque rescatados, guardan relación con aquellos aprendizajes de la niñez. Cursos que se suceden, ofertas que se imponen con formatos cerrados y se interrumpen o superponen con otras son algunas de las facetas que abonan una idea de excepcionalidad que dificulta pensar el largo plazo y a la continuidad.

En el plano subjetivo, la sucesión ininterrumpida de propuestas formativas focalizadas, cortoplacistas y sin articulación entre sí contiene una idea de largo plazo que, aunque no está explícita, se hace presente. Y es la idea de un eterno aprendiz que es 
interpelado por cada nueva propuesta desde el saber del que carece, mas nunca por aquello de lo que dispone.

La identificación, la ponderación, la enumeración son acciones que se desprenden de la intención del mapeo de saberes. Hacerlos inteligibles, concretos, visibles son acciones embrionarias en un camino potencialmente capaz de contribuir a generar pertenencia. El mapeo conduce a una multiplicidad de acciones concomitantes: comparar para mensurar, asociar para comprender y pasar en limpio para valorar. Cada una de estas hace imprescindible una lectura en el marco de las experiencias que las generan.

Por último, el mapeo registra, además de saberes individuales, una experiencia colectiva que, por emergente, promueve el interés en su indagación: los espacios formativos entre pares generados y motorizados por los propios detenidos.

Alguien que tiene un saber, unos compañeros que son convocados a aprender, una logística que apunta a la provisión de insumos y herramientas y otra destinada a la comercialización generan un escenario concreto de proyecto productivo con algunos componentes de taller autogestionado, en la tipología antes aludida.

\section{Algunas conclusiones}

El trabajo es un eslabón más de las sucesivas e inagotables exclusiones de las personas detenidas. También lo son la educación, la salud y gran parte del entramado de instituciones cuya misión es garantizar el acceso a los derechos y que tiene como horizonte el desarrollo integral de la persona. Paradójicamente, el ingreso a la cárcel tiene el registro, en la trayectoria vital de estos sujetos, de una experiencia novel en materia de inserción o inclusión social.

En este sentido, y partiendo del reconocimiento del carácter rupturista con que la universidad despliega su inserción en los espacios de la cárcel, reivindicamos la tarea de hacer inteligibles los saberes que estos sujetos tienen. Si lo antedicho es fundante para entender el trabajo como derecho humano, ocuparse de hacer perceptible la dimensión temporal con que se aprende y se trabaja permitirá iniciar un camino hacia la autogestión. Y esta tiene como condición necesaria la transmisión y socialización de saberes como un área indelegable de sus protagonistas. Con acompañamientos varios, pero sin el tutelaje de nadie.

La lógica carcelaria habrá de cargar al trabajo con sentidos que subrayan la supervivencia, el logro rápido de recursos, la inmediatez de las necesidades, y todo ello oficia de gran conspirador para pensar el trabajo como un derecho y la autogestión como estructurante de otras visiones de sí mismo. La cárcel no hace más que prolongar este encadenamiento que prevalece en las historias de estos sujetos. Los proyectos no tienen 
continuidad, son reemplazados por explicaciones externas o sirven para suplir las demandas locales de mano de obra.

En este sentido, la prescindencia de la dimensión temporal con la que se significa el trabajo en la cárcel aparece como la mayor amenaza para la continuidad de los proyectos productivos de los detenidos, y una verdadera falacia para los proyectos asociativos. La proyección a largo plazo de los emprendimientos obliga a pensar en etapas, conduce a marcar objetivos, a socializar responsabilidades y a establecer relaciones con el afuera y el adentro, pero no de la cárcel, sino del emprendimiento y de las personas que lo integran. En este marco, la culminación de la condena no ha de ser una encrucijada que atenta contra el proyecto. Su extensión, tampoco.

Por último, corresponde señalar los desafíos que supone potenciar una concepción del trabajo autogestivo que ordene y oriente las acciones del trabajo en la cárcel y confiera sentido estratégico a las experiencias novedosas en marcha. La consecución de proyectos asociativos y el carácter autogestivo que emerge de ellas tiene como condición necesaria el deslizamiento de sentidos dominantes. Es una condición necesaria, pero no suficiente. La profundidad del cambio al que aludimos supone una incidencia capaz de ingresar y permear las prácticas y los imaginarios, y esto amerita un compromiso que trascienda los espacios universitarios e involucre a gran parte del entramado institucional del Estado. La capilaridad con que actúa la lógica carcelaria requiere un accionar igual de organizado y extendido y equivalente en su sistematicidad.

En consecuencia, la permanencia de espacios de autogestión no habrá de lograrse sin el compromiso de fortalecer las relaciones interinstitucionales de las organizaciones que conviven y están implicadas en la construcción cotidiana de la cárcel. En este plano, resulta imperioso asumir la importancia estratégica de la institucionalización de las prácticas, de los saberes producidos, las estrategias implementadas, los logros obtenidos y los acuerdos a los que se arribó.

Por último, conviene subrayar la importancia de la sistematización de las experiencias, de modo que conduzca a las organizaciones a hacer inteligibles los saberes producidos. Esta cuestión, que venimos trabajando (Sosa, 2012; 2013), resulta imprescindible no solo en las disputas de sentido cotidianas, sino, sobre todo, en la potencialidad que comienza a ser advertida en los procesos de reflexividad de la experiencia. La lucha cultural que implican las experiencias alternativas no solo ha de ser con los otros, sino sobre todo con nosotros mismos, en cuanto sujetos que las protagonizan y llevan adelante. En este plano se inscriben los desafíos que implica la construcción de una sociedad que promueva espacios en los que las personas se constituyan como sujetos de derecho. 


\section{Referencias bibliográficas}

Abravanel, H. (rev). (1992). Cultura organizacional: Aspectos teóricos, prácticos y metodológicos. Bogotá: Legis.

Argentina. (1999). Ley 12.256: Ley de Ejecución Penal. Recuperado de https:// intranet.hcdiputados-ba.gov.ar/refleg/lw12256.pdf.

Baczko, B. (1991). Los imaginarios sociales: Memorias y esperanzas colectivas. Buenos Aires: Nueva Visión.

Barbier, J. (dir.) (1996). Saberes teóricos y saberes de la acción. París: PUF.

Carton, M. (1985). La educación y el mundo del trabajo. Ginebra: UNESCO.

Guber, R. (1991). El salvaje metropolitano: A la vuelta de la antropología postmoderna. Reconstrucción del conocimiento social en el trabajo de campo. Buenos Aires: Legasa. Habermas, J. (1987). Teoría de la acción comunicativa. Tomo 2. Madrid: Taurus.

Lourau, R. (1988). El análisis institucional. Buenos Aires: Amorrortu.

Marx, C. (1973). Elementos fundamentales para la crítica de la economía política (borrador), 1857-1858. Buenos Aires: Siglo XXI.

Ministerio de Justicia y Derechos Humanos. (2018). Informe anual del Sistema Nacional de Estadísticas sobre Ejecución de la pena. Recuperado de http://www.saij. gob.ar/docs-f/estadisticas-sneep/2018/InformeSNEEPARGENTINA2018.pdf.

Ministerio de Justicia y Derechos Humanos. (2019). Sistema Nacional de Estadísticas sobre Ejecución de la Pena (SNEEP). Recuperado de http://datos.jus.gob.ar/ ro/dataset/sneep.

Sosa, R. (2012). La construcción de «otra economía»: Los sentidos de educar en una experiencia de finanzas solidarias. Otra Economía, 6(11), 198-210. doi: 10.4013/otra.2012.611.09.

Sosa, R. (2013). La educación que necesitamos en Olavarría: Sentidos e imaginarios en la definición de prioridades educativas de una ciudad de rango medio. En La antropología social hoy: A 10 años del nuevo siglo. Trabajo presentado en las IV Jornadas de Antropología Social del Centro, Buenos Aires.

Sosa, R. (2014). La marca en el orillo: Prioridades educativas, imaginarios urbanos y perfil productivo de la ciudad de Olavarría. (Tesis de doctorado). Buenos Aires: Universidad de Buenos Aires.

Umpiérrez, A. (2013). En la educación hay muchas educaciones posibles. Recuperado de https://www.unicen.edu.ar/content/en-la-educaci\%C3\%B3n-hay-muchas-educaciones-posibles.

Verón, E. (1987). La semiosis social. Barcelona: Gedisa.

Williams R. (1982). Cultura, sociología de la comunicación y del arte. Buenos Aires: Paidós. 